University of Nebraska - Lincoln

DigitalCommons@University of Nebraska - Lincoln

Faculty Publications from the Department of Electrical \& Computer Engineering, Department Electrical and Computer Engineering

2011

\title{
Wide-Area Multi-Object Tracking With Non-Overlapping Camera
}

\section{Views}

Youlu Wang

University of Nebraska-Lincoln, youlu.wang@huskers.unl.edu

Senem Velipasalar

University of Nebraska-Lincoln, velipasa@engr.unl.edu

M. Cenk Gursoy

University of Nebraska-Lincoln, gursoy@engr.unl.edu

Follow this and additional works at: https://digitalcommons.unl.edu/electricalengineeringfacpub

Part of the Electrical and Computer Engineering Commons

Wang, Youlu; Velipasalar, Senem; and Cenk Gursoy, M., "Wide-Area Multi-Object Tracking With NonOverlapping Camera Views" (2011). Faculty Publications from the Department of Electrical and Computer Engineering. 212.

https://digitalcommons.unl.edu/electricalengineeringfacpub/212

This Article is brought to you for free and open access by the Electrical \& Computer Engineering, Department of at DigitalCommons@University of Nebraska - Lincoln. It has been accepted for inclusion in Faculty Publications from the Department of Electrical and Computer Engineering by an authorized administrator of DigitalCommons@University of Nebraska - Lincoln. 


\title{
WIDE-AREA MULTI-OBJECT TRACKING WITH NON-OVERLAPPING CAMERA VIEWS
}

\author{
Youlu Wang, Senem Velipasalar, Mustafa Cenk Gursoy \\ University of Nebraska-Lincoln \\ Dept. of Electrical Engineering, 209N SEC, Lincoln, NE, 68588 \\ youlu.wang@huskers.unl.edu, velipasa@engr.unl.edu,gursoy@engr.unl.edu
}

\begin{abstract}
We present a system for wide-area multi-object tracking across disjoint camera views. We employ a probabilistic Petri Net-based approach to account for the uncertainties of the vision algorithms (such as unreliable background subtraction, and tracking failure) and to incorporate the available domain knowledge. We combine appearance features of objects as well as the travel-time evidence for target matching and consistent labeling across disjoint camera views. 3D color histogram, Histogram of Oriented Gradients, object size and aspect ratio are used as the appearance features. The distribution of the travel time is modeled by a Gaussian Mixture Model. By incorporating the domain knowledge about the camera configurations and the information about the received packets from other cameras, certain transitions are fired in the probabilistic Petri net. The system is trained to learn different parameters of the matching process. We present widearea tracking of vehicles as an example where we used three non-overlapping cameras. The first and the third cameras are approximately 150 meters apart from each other with two intersections in the blind region. The results show the success of the proposed method.
\end{abstract}

Index Terms - Object Tracking, Non-overlapping Views, Probabilistic Petri-Nets

\section{INTRODUCTION}

Multi-camera systems are widely used for video surveillance, traffic monitoring, statistics gathering, and commercial, military and public transportation applications. In a multi-camera setup, cameras should collaborate with each other to track objects consistently for longer periods of time. In wide-area tracking and wide-area surveillance applications, it is not always realistic to assume that all the cameras in the system will have overlapping fields of view. For instance, in airports there are many outdoor and indoor cameras, and their views do not overlap. Thus, it is very important to have algorithms for tracking targets across non-overlapping cameras, and this topic has attracted much interest in recent years. Tracking across disjoint camera views is a more challenging problem

This work was supported partly by the National Science Foundation under grants CNS 0834753 and CCF-0917265. than tracking with overlapping cameras due to lack of spatial continuity, and thus having blind regions.

Appearance is an obvious feature to associate objects in different camera views. However, performance of the methods using only object appearance may easily be influenced by the illumination variations, different camera parameters and different camera angles. To make use of the spatial features, similar to what has been used for the overlapping cameras [1], methods have been introduced to predict the object's motion by assuming a common ground plane and small gap between two cameras [2, 3]. Kang et al. [2] use a spatio-temporal Joint Probability Data Association Filter (JPDAF) to formulate a joint probability model encoding objects' appearance and motion. Two non-overlapping camera views are warped in the reference of a moving camera view and merged into a mosaic. Thus, the object's motion can be inferred when it's in the gap between two stationary cameras. Chilgunde et al. [3] use a Kalman Filter to predict the object's location in the small gap between two camera's view. Javed et al. [4] propose a system using a brightness transfer function as well as a space-time pdf including velocity and travel time. The best match will be found by maximizing the a posteriori probability. Huang et al. [5] use the transition time as the only evidence to infer the traffic flow status across non-overlapping views. A Gaussian Mixture Model (GMM) of the transition time is built without identifying the object correspondences explicitly. Several other studies have focused on recovering the topology of a camera network consisting of cameras with disjoint views. Niu et al. [6] use the appearance model to measure the similarity between disappearing and reappearing trajectories, then detect the possible link between the disjoint views, and estimate the transition time by the weighted cross correlated model. Finally, the non-overlapping network topology is recovered based on the estimated mutual information. Makris et al. [7] use a graphical model to represent the probabilistic links between the camera nodes. The topology of the camera views is determined by the set of the valid links and their transition times.

We propose a system for wide-area tracking across disjoint camera views. We employ a probabilistic Petri Net $(\mathrm{pPN})$-based approach to account for the uncertainties of the vision algorithms (such as unreliable background subtraction, 
tracking failure, object matching confidence) and to incorporate domain knowledge. We combine appearance features of objects as well as the travel-time evidence in a Matching Box for consistent target labeling across disjoint camera views. 3D color histogram, Histogram of Oriented Gradients (HOG), object size and aspect ratio are used as the appearance features. The distribution of the travel time is modeled by a Gaussian Mixture Model (GMM) and estimated by the EM algorithm. By incorporating the domain knowledge about the camera configurations and the information about the received packets from other cameras, certain transitions are fired in the pPN. The similarity score output of the Matching Box is used as the probability of transition in the pPN. The system is trained to learn different parameters of the Matching Box.

We also use domain knowledge when sending packets about the objects leaving a camera's view. For instance, depending on the road, a vehicle's lane can be used to determine whether it is going to turn left/right or go straight. A camera needs to communicate only with its immediate neighbors, which makes our system scalable.

The main contributions of this work are as follows: 1) the domain knowledge and the uncertainties are accounted for and incorporated into a pPN; 2) object matching across nonoverlapping views employs multiple appearance features; 3 ) appearance features are combined with the GMM model of travel-time to increase robustness. The proposed approach can easily be generalized to various surveillance applications involving disjoint camera views, such as indoor human tracking or outdoor human/vehicle tracking. In this paper, widearea tracking of vehicles is presented as an example. Similar approach together with a different set of domain knowledge can be employed for tracking people in a disjoint camera setup. For example, for indoor people tracking, the same set of features presented here can be employed. However, a camera may save the information of leaving people for a while, since a person may leave its view and come back later instead of moving towards the next camera. Thus, in addition to the candidates that are received from the previous camera(s), the objects that left before should also be considered as candidates. The two different types of candidates should have different sets of weights for appearance features. For instance, for the objects leaving the view and coming back, the travel time should have lower weight, since the return time of a person might have high variance.

\section{A PETRI NET-BASED APPROACH FOR TRACKING AND OBJECT MATCHING}

A Petri Net (PN) is used for modeling the relations between the conditions and events in dynamic systems [8, 9]. It is a particular type of directed bipartite graph composed of places and transitions. The places and the transitions are connected by arcs, where input arcs are from a place to a transition and output arcs are from a transition to a place. The places contain the tokens. Transitions are active components. If there are enough tokens in the input places, the transitions are enabled. Transitions are only allowed to fire if they are enabled. When a transition in enabled and the condition associated to this transition is satisfied, it fires. When the transition fires, it removes tokens from its input places to its output places.

There have been various extensions of the Petri nets such as Colored PNs, Continuous PNs, Stochastic timed PNs and Fuzzy PNs. Albanese et al. [10] proposed the probabilistic Petri net $(\mathrm{pPN})$ for modeling the uncertainty and inaccuracies in a video surveillance system. Compared to the original PNs, a probability is attached to every arc pointing from a place to a transition in the pPN. A token is assigned the probability 1 at the initial place. When it moves to the next place, the probability is multiplied by the probability attached to the arc. After moving through the whole PN from the initial place to the end place, the final probability is the product of the probabilities attached to the arcs through which the token has passed.

We adopt a pPN-based approach to perform object tracking and consistent labeling on a camera. Figure 1 shows the graphical model of the steps employed by camera 3 in a threecamera setup. The camera configuration can be seen on the upper left-hand corner of the Fig.1. In this model, the uncertainties and inaccuracies could be created by the background subtraction, the tracking algorithm or the object matching process, and are modeled by probabilities $p_{b}, p_{t}$, and $p_{m}$, respectively. We include a Tracking Box in the model, which represents the tracking algorithm used by the camera. As mentioned before, a Matching Box is used to represent the object matching process using multiple features. In Fig. 1, an arc with no probability on it means that its probability is assumed to be 1 .

Once a new object is detected, it will be put in the START place $l_{0}$. Then, it immediately moves into $l_{1}$, since there is no condition attached to $t_{0}$. From $l_{1}$ to $t_{1}$, a probability $p_{b}$ is attached to the arc to model the reliability of the background subtraction. This probability is learned during training. Then, the object is moved into the Tracking Box, where a tracker for this object is created and updated every frame until it leaves the camera's view. If the object enters the view from side $S$ and there are received packets from the neighboring camera(s) on this side, this object will be assigned a temporary label first, and moved into the Matching Box in an attempt to find a match from the received candidates which had left the view of the neighboring camera(s). A probability $p_{m}$ will be attached to the token as the output of the Matching Box. If no candidate package has been received from other camera(s), a new label will be assigned. The tracking process is performed every frame and the probability $p_{t}$ indicating the tracking confidence is updated every frame until the object leaves the camera's view. Thus, for an object tracked by the system, the final probability of the tracking is:

$$
p=p_{b} \cdot p_{m} \cdot p_{t}
$$

The details of the Tracking Box and the Matching Box will be described in Sec.3 and Sec.4, respectively. 


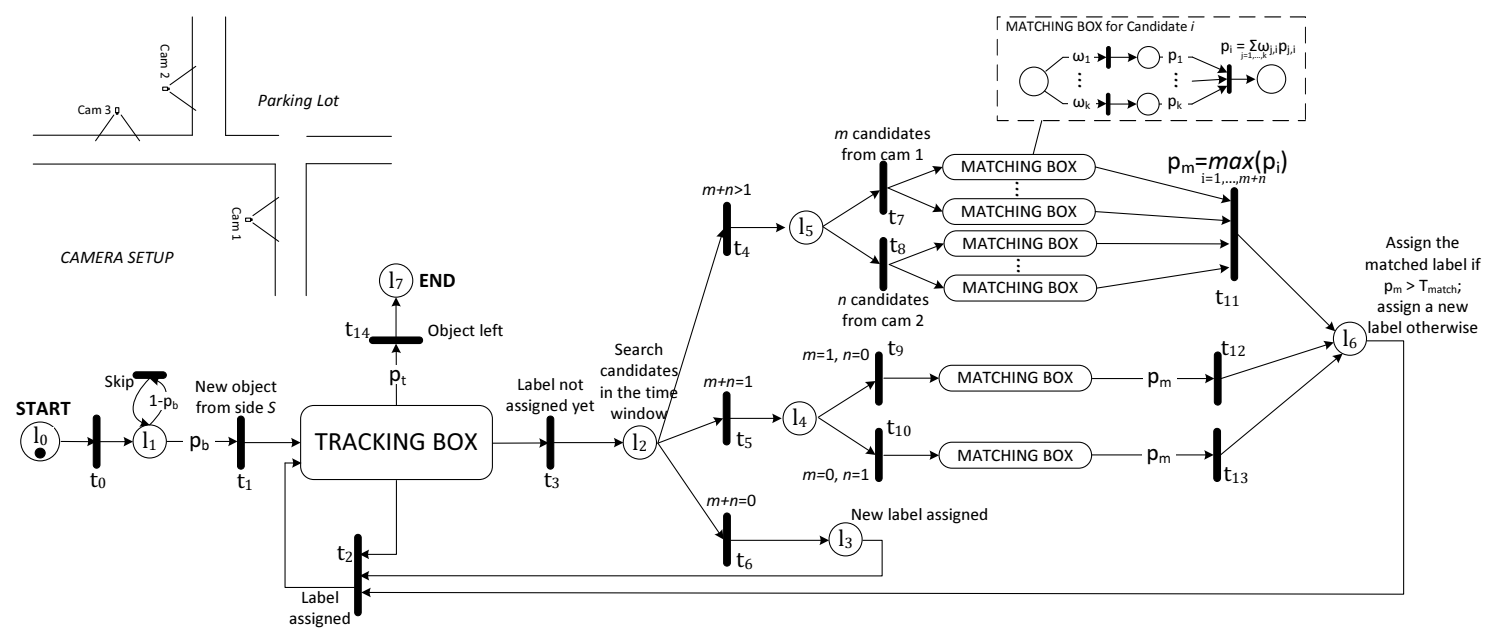

Fig. 1. Probabilistic Petri Net for tracking and object matching.

The camera setup for our system is shown on the upper left-hand corner of Fig. 1. Example images captured by these three cameras can be seen in Fig. 4. Camera 3 is watching a one-way road. When a vehicle enters into the view of Camera 3, it may have come from the view of Camera 1, view of Camera 2, or from regions that are not watched by any cameras. In the latter case, camera 3 needs to detect this correctly, and assign a new label to the vehicle.

\section{THE TRACKING ALGORITHM}

For every incoming frame, foreground detection is performed by using a robust and lightweight algorithm that is presented in [11]. Then, foreground pixels are grouped into blobs by connected component labeling. Each blob corresponds to a detected object. In our traffic application example, lane markers provide useful information to remove the noise pixels and uninteresting foreground objects. When a new foreground blob is detected inside the lanes, a new tracker is created. The label of this tracker, the coordinates of the bounding box and the color histogram are saved in the tracker. Each bin in the 3-D histogram corresponds to an $(\mathrm{R}, \mathrm{G}, \mathrm{B})$ range.

In [12], a P2P multi-camera system is presented wherein each camera is attached to a different CPU and cameras have partially overlapping fields of view. For tracking on a single camera view, we use an optimized version of the tracking algorithm proposed in [12]. At every frame, trackers are matched to foreground blobs by using a matching criteria based on the bounding box intersection and the Bhattacharyya coefficient [13] that is derived from the sample data by using:

$$
\hat{\rho}(y) \equiv \rho[\hat{\mathbf{p}}(\mathbf{y}), \hat{\mathbf{q}}]=\sum_{u=1}^{m} \sqrt{\hat{p}_{u}(\mathbf{y}), \hat{q}_{u}}
$$

where $\hat{\mathbf{q}}=\left\{\hat{q}_{u}\right\}_{u=1 \ldots m}$, and $\hat{\mathbf{p}}(\mathbf{y})=\left\{\hat{p}_{u}(\mathbf{y})\right\}_{u=1 \ldots m}$ are the probabilities estimated from the $\mathrm{m}$-bin histogram of the model in the tracker and the candidate blobs, respectively. If the bounding box of a foreground blob intersects with that of the current model mask of the tracker, the Bhattacharyya coefficient between the model histogram of the tracker and the histogram of the foreground blob is calculated by using (2). The tracker is assigned to the blob which results in the highest Bhattacharyya coefficient. The Bhattacharyya coefficient with which the tracker is matched to its object is called the similarity coefficient.

A tracking score $p_{t}$ is calculated and updated for every tracker to account for the errors that may be caused by segmented objects and unresolved merges/splits. $p_{t}$ is the output of the Tracking Box, and is the product of the average similarity coefficient and a confidence measure. The confidence measure is based on the length of the trajectory. It is the ratio between the current trajectory length and the length of the road in the view. When the object leaves the view, the confidence measure of the trajectory length approaches 1 .

\section{OBJECT MATCHING ACROSS NON-OVERLAPPING VIEWS}

When an object leaves a camera's view, this camera creates and sends a message packet containing the appearance features, exit time and the label of this object. Appearance features, travel-time evidence and the matching procedure are described in detail below.

\subsection{Color Histogram}

As mentioned above, we use 3D color histogram of the detected foreground objects as one of the appearance features. Each bin in the histogram corresponds to an $(\mathrm{R}, \mathrm{G}, \mathrm{B})$ range. The similarity score $s_{C H}$ between two histograms is calculated by Eq. (2).

\subsection{Texture}

Texture is a useful feature for object detection or tracking. In [14], Histogram of Oriented Gradients (HOG) descriptor is proposed for human detection. The local object textures are modeled by calculating the distribution of the local intensity gradients and the edge directions.

A foreground blob is divided into $n$ cells. For each cell, an $m$-bin HOG is built. Each bin in the HOG corresponds to 


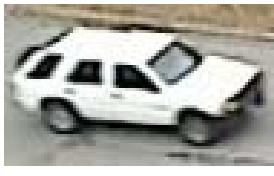

(a) The object

(b) Foreground pixels

(c) Rotated pixels

Fig. 2. Example of the angle correction for HOG descriptor.

an orientation spanning. The combination of $n$ HOG forms the HOG descriptor, with the size of $m * n$ bins. To calculate the HOG, the intensity gradients are calculated first in both the horizontal and vertical directions. Then, the magnitude and the orientation of the gradient is calculated. According to the discussion in [14], unsigned orientations spanning from 0 to 180 degree in conjunction with 9 histogram bins perform best. Each gradient has a vote in its bin, which is its magnitude. An important step described in [14] is the local normalization, which helps to reduce the impacts of the illumination and contrast variations. The cells are grouped into blocks and block-based normalization is performed. The blocks are partially overlapped with each other, thus each cell contributes more than once to the final descriptor.

The similarity $s_{T}$ between two HOG descriptors $A$ and $B$ is calculated by Cosine Similarity:

$$
s_{T}=\cos (\theta)=\frac{A \times B}{\|A\|\|B\|}
$$

One factor that influences the HOG descriptor is the different camera angles in different views. To address this problem in vehicle tracking, we rotate the foreground pixels by the angle of the lane before building the HOG descriptor. Figure 2 shows an example of the angle correction. Figures 2(a) and (b) show the color image of the object and the segmented foreground pixels, respectively. The bounding box is also drawn along the direction of the lane in Fig. 2(b). If the angle between the lane and $x$-axis is denoted by $\theta$, the foreground image (Fig. 2(b)) is warped by the rotation matrix

$$
\left[\begin{array}{cc}
\cos \theta & -\sin \theta \\
\sin \theta & \cos \theta
\end{array}\right] .
$$

Figure 2 (c) shows the rotated foreground pixels.

\subsection{Aspect Ratio and Size}

The aspect ratio is a useful feature that can be used to differentiate the compact cars and large-sized vehicles in a traffic application. If the aspect ratios of the two objects are $a_{1}$ and $a_{2}$ respectively, the similarity score is calculated by

$$
s_{A}=1-\left|\frac{a_{1}-a_{2}}{a_{1}}\right|
$$

The object size is also used as an appearance feature. It is the number of pixels in the detected foreground blob. The size of an object varies depending on its distance from the camera. Figure 3 shows an example, where the cars coming from Camera 1 travel in different lanes in the view of Camera 3 . Let the sizes of the two objects be $s_{1}$ and $s_{2}$ in the previous

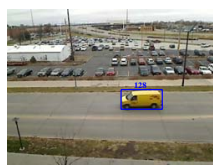

(a) Camera 1

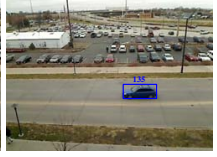

(b) Camera 1

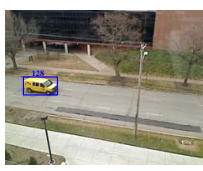

(c) Camera 3

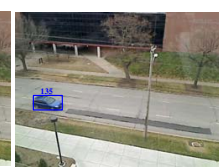

(d) Camera 3
Fig. 3. Example of the different size factors of the cars in different lanes.

camera and current camera, respectively. Also, let $f_{l}$ denote the size ratio of the objects, i.e. $f_{l}=s_{1} / s_{2}$. $f_{l}$ will be different depending on the lane the vehicle is traveling in. The closer the object is to the camera, the bigger $s_{2}$ is, and thus the smaller $f_{l}$ is. The $f_{l}$ for different lanes are learned during training. The similarity score is then calculated by

$$
s_{S}=1-\left|\frac{s_{1}-s_{2} * f_{l}}{s_{1}}\right| \text {. }
$$

\subsection{Travel Times}

In traffic flow, travel times of the cars across the blind regions are dependent on the traffic conditions and traffic lights. We use a Gaussian Mixture Model (GMM) to model the distribution of the travel times in the blind region. The travel time of a car is the difference between the time the car enters the current camera view and the time the car exits the previous camera view. The parameters of the GMM are estimated in the training stage by using a standard Expectation-Maximization(EM) algorithm. These parameters are the weight $\omega$, and the mean $\mu$ and the variance $\sigma^{2}$ for each Gaussian distribution.

For calculating the similarity score of the travel times between a candidate and detected object, the travel time $t$ is obtained by

$$
t=t_{o, a}-t_{c, l}
$$

where $t_{o, a}$ is the time the object enters the current camera view, and $t_{c, l}$ is the time the candidate leaves the previous camera view. Similar to the clustering or classification problems that adopt a GMM, a Gaussian distribution that yields the highest probability is selected as the distribution that this time value belongs to. Considering the various factors that may influence the travel time, a travel time that falls into the range of mean \pm one standard deviation does not get penalized, i.e. it will have a score of 1 . Otherwise, the similarity score of travel time is calculated by

$$
s_{T T}=e^{-\frac{(x-\mu)^{2}}{2 \sigma^{2}}} / e^{-\frac{(\mu+\sigma-\mu)^{2}}{2 \sigma^{2}}}=e^{-\frac{(x-\mu)^{2}-\sigma^{2}}{2 \sigma^{2}}}
$$

where $\mu$ and $\sigma$ are the mean and the standard deviation of the Gaussian distribution component that this object belongs to.

\subsection{Weighted Matching Criteria}

When a new object enters into the view of the current camera from side $S$, it first checks if there are candidate packages sent by the neighboring camera(s) on side $S$. If there are, the current camera tries to find a match among the $C$ received candidates. Each candidate, $i \in\{1 \ldots C\}$, has different features that are described above, namely color histogram $(\mathrm{CH})$, 
texture (T), aspect ratio (A), size (S) and travel time (TT). For each feature $j \in F=\{C H, T, A, S, T T\}$, a similarity score $s_{j}^{i}$ is calculated and is given a weight $w_{j}$. To combine multiple features, an overall similarity score is calculated. The best matching candidate object $O$ is found by

$$
O=\arg \max _{i \in\{1 \ldots C\}} \sum_{i \in\{1 \ldots C\}, j \in F}\left(w_{j} s_{j}^{i}\right) .
$$

If the overall similarity score of the object $O$ is greater than a pre-defined threshold, then the candidate object $O$ is matched to this tracker, and the tracker is assigned the label of $O$. The overall similarity score is shown as $p_{m}$ in Fig. 1. Otherwise, a new label is assigned to this tracker.

\section{EXPERIMENTAL RESULTS}

We performed the wide-area vehicle tracking experiments with three disjoint cameras having the configuration shown in Figure 1. We used 3 hours of video data for training, and another 15 minutes of video data from three cameras for testing. Figure 4 shows the views of the three cameras. In Camera 1 and Camera 2, the viewed roads are two-way, but only the direction in which a car can travel towards Camera 3 was considered. Camera 3, on the other hand, watches a one-way road. The cars entering into the view of Camera 3 may come from Camera 1, Camera 2 or other blind regions that are not watched by any other camera. The distance between Camera 1 and Camera 3 is approximately 150 meters, with two intersections in the blind region. One of the intersections, which is close to Camera 2, has traffic lights. These intersections influence the travel time in the blind region vary significantly. The distance between Camera 2 and Camera 3 is approximately 20 meters. The cars that wait for the green light can still be seen in Camera 2's view. Thus, this intersection does not impact the travel time between Camera 2 and Camera 3 .

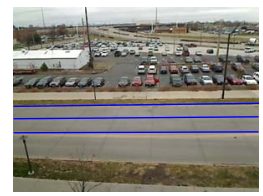

(a) Camera 1

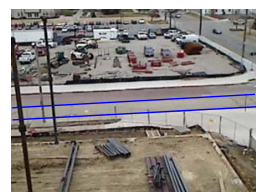

(b) Camera 2



(c) Camera 3
Fig. 4. Views of the three cameras.

\subsection{Training Stage}

\subsubsection{Domain Knowledge}

The first domain knowledge that should be learned during training is the camera configuration. In Camera 1's view, as shown in Figure 4 (a), there are two lanes, and the view is close to the intersection. Based on the traffic rules, normally the cars that will turn left/right will move to the left/right lane. Thus, by detecting lanes, the cars on the right lane can be removed from the candidate list to be sent out.

Since there are two intersections in the blind region and one of them has the traffic lights, there are more than one possible distributions to represent the travel times. Thus, a GMM is built to model the travel times of the cars traveling from Camera 1's view to Camera 3's view. In our experiments, a GMM with three mixtures is trained. The means, variations and weights for each Gaussian distribution are $(22.83,24.30,0.464),(39.37,53.26,0.427)$ and $(60.36,123.92,0.109)$, respectively. The plot of the GMM is shown in Figure 5.

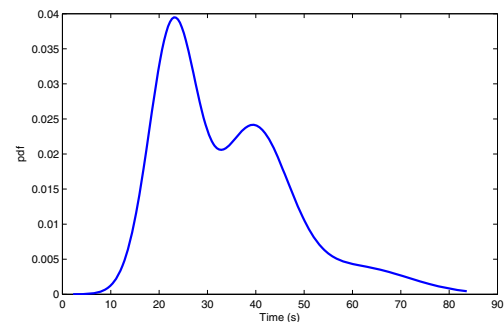

Fig. 5. Gaussian Mixture Model of the Travel Time between Camera 1 and Camera 3.

Camera 2 watches only one lane. The cars on the lane may turn left or right. Since the cars are still in the view when they wait for the green light, there is no need to build a GMM for the travel times. Thus, a time window is picked to eliminate the candidates that fall outside this window.

Also, the angles of the lanes with respect to the $\mathrm{x}$ axis are learned in the training stage. This is used for correcting the HOG descriptors.

\subsubsection{Uncertainty}

The reliability of background subtraction, $p_{b}$, is $0.997 . p_{t}$ and $p_{m}$ are be calculated in the tracking box and the matching box for each object during the testing stage.

\subsection{Testing Stage}

In the testing stage, the weights for the color, texture, travel time, size and aspect ratio are set to be $0.35,0.25,0.2,0.05$ and 0.15 respectively. The threshold for the overall similarity score is 0.77 . In Camera 1 , the cars leaving the view from the right lane are not sent out as candidates to Camera 3. There are 34 candidate vehicles detected and sent out by Camera 1. In Camera 2, 18 candidate vehicles are detected and sent out. Camera 3 detects 55 cars entering its view from left. Among these 55 cars, 50 of them are assigned correct labels after the matching process, and a success rate of $90.91 \%$ is achieved. Our algorithms run on a PC with $2.13-\mathrm{GHz}$ Intel Core Duo processor and 4GB memory. It takes $31 \mathrm{~ms}$ to perform background subtraction; and $16 \sim 32 \mathrm{~ms}$ for tracking algorithm, including feature extraction and object matching.

Figure 6 shows an example where three cars enter the view of Camera 3 consecutively. Object 112 came from Camera 1, Object 202 came from Camera 2 and Object 303 came from the blind region (i.e. received a new label). They are all assigned correct labels. When the red car entered the view of Camera 3 as seen in Fig. 6(c), there were two candidates received from Camera 1 and one candidate from Camera 2. After going through the matching box, the maximum matching score was smaller than the matching threshold 0.77 . Thus, 


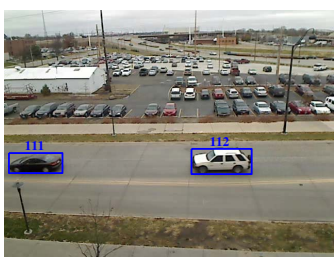

(a) Camera 1

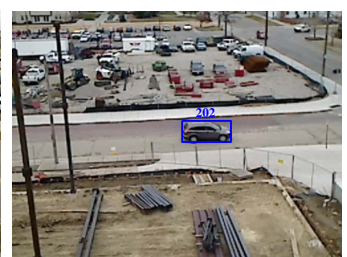

(b) Camera 2

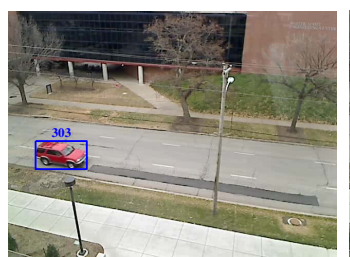

(c) Camera 3

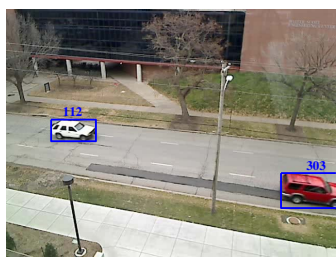

(d) Camera 3

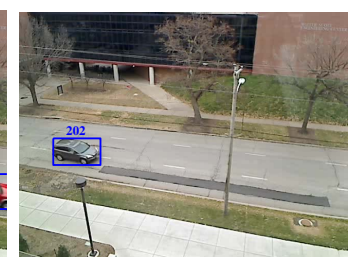

(e) Camera 3

Fig. 6. Example of matched cars.

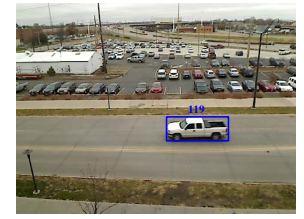

(a) Camera 1

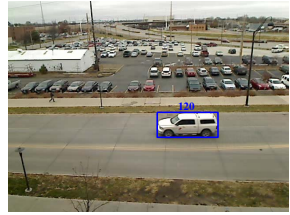

(b) Camera 1

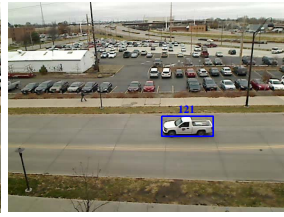

(c) Camera 1

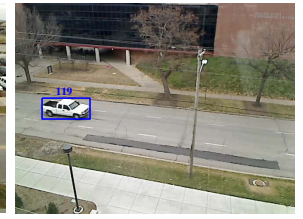

(d) Camera 3

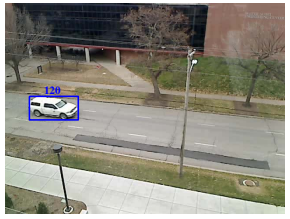

(e) Camera 3



(f) Camera 3

Fig. 7. Example of matched cars with similar features.

this car is assigned a new label 303. In Fig. 6(d), a white car enters the scene. There were four received candidate packages in total, three from Camera 1 and one from Camera 2. After the matching process, the candidate with the label 112 has the maximum matching score of 0.8218 , and the white car is assigned the correct label 112. The third car in Fig. 6(e) were compared with the same four candidates. The candidate with the label 202 resulted in the highest matching score of 0.8742 , and the car was given the correct label of 202 .

Figure 7 shows a more challenging example where three consecutive cars with the same color and similar texture enter the view of camera 3 . The cars are matched to the correct candidates with matching scores of $0.8299,0.8507$ and 0.8144 , respectively. The matching process performs well even if the objects have similar appearances.

\section{CONCLUSION}

We have presented a wide-area multi-object tracking system composed of non-overlapping cameras. A probabilistic Petri Net-based approach has been used to account for the uncertainties of the vision algorithms and to incorporate the available domain knowledge. Multiple features are used for object matching across non-overlapping views, including $3 \mathrm{D}$ color histograms, HOG descriptors, object sizes, aspect ratios and travel times. The distribution of the travel time has been modeled by a Gaussian Mixture Model. We have presented widearea tracking of vehicles as an example where we used three non-overlapping cameras. Based on the 55 cars entering the last camera's view, a success rate of $90.91 \%$ was achieved in terms of correctly labeling them.

\section{REFERENCES}

[1] N. Anjum and A. Cavallaro, "Trajectory association and fusion across partially overlapping cameras," Proc. of the IEEE Intl Conf. on Advanced Video and Signal Based Surveillance.

[2] J. Kang, I. Cohan, and G. Medioni, "Persistent objects tracking across multiple non overlapping cameras," Proc. of the IEEE Workshop on Motion and Video Computing, 2005.
[3] A. Chilgunde et al., "Multi-camera target tracking in blind regions of cameras with non-overlapping fields of view," Proc. of the British Machine Vision Conference, 2004.

[4] O. Javed, K. Shafique, and M. Shah, "Appearance modeling for tracking in multiple non-overlapping cameras," Proc. of IEEE Conf. on Computer Vision and Pattern Recognition, 2005.

[5] C.C. Huang, W.C. Chiu, S.J. Wang, and J.H. Chuang, "Probabilistic modeling of dynamic traffic flow across nonoverlapping camera views," Proc. of the Int'l Conf. on Pattern Recognition, 2010.

[6] C. Niu and E. Grimson, "Recovering non-overlapping network topology using far-field vehicle tracking data," Proc. of the Int'l Conf. on Pattern Recognition, 2006.

[7] D. Makis, T. Ellis, and J. Black, "Bridging the gaps between cameras," Proc. of IEEE Computer Society Conf. on Computer Vision and Pattern Recognition, 2004.

[8] R. David and H. Alla, "Petri nets for modeling of dynamic systems," Automatica, vol. 30, no. 2, pp. 175-202.

[9] T. Murata, "Petri nets: Properties, analysis and applications," Proc. of the IEEE, vol. 77, no. 4, pp. 541-580.

[10] M. Albanese, R. Chellappa, V. Moscato, and A. Picariello, "A constrained probabilistic petri net framework for human activity detection in video," IEEE. Trans. on Multimedia, vol. 10, no. 8, pp. 1429-1443, 2008.

[11] M. Casares, S. Velipasalar, and A. Pinto, "Light-weight salient foreground detection for embedded smart cameras," Computer Vision and Image Understanding, vol. 114, no. 11, pp. 12231237, 2010.

[12] S. Velipasalar et al., "A scalable clustered camera system for multiple object tracking," EURASIP Journal on Image and Video Processing, vol. 542808, 2008.

[13] D. Comaniciu, V. Ramesh, and P. Meer, "Real-time tracking of non-rigid objects using mean shift," Proc. of IEEE Conf. on Computer Vision and Pattern Recognition, pp. 142-149, 2000.

[14] N. Dalal and B. Triggs, "Histograms of oriented gradients for human detection," Proc. of IEEE Computer Society Conf. on Computer Vision and Pattern Recognition, vol. 1, pp. 886-893. 